\title{
Disk Diffusion Method
}

National Cancer Institute

\section{Source}

National Cancer Institute. Disk Diffusion Method. NCI Thesaurus. Code C85595.

A method to determine microbial susceptibility to antibiotics in which filter paper disks containing known concentrations of antibiotics are placed on the agar surface that has been previously inoculated with the bacteria of interest. Microbial susceptibility is assessed by measuring the area around the disk that is free of microbial growth. 